UWThPh-2001-45

WUE-ITP-2001-034

hep-ph/0111285

\title{
Production of singlino dominated neutralinos in extended supersymmetric models
}

\author{
F. Franke丹 \\ Institut für Theoretische Physik und Astrophysik, Universität Würzburg, \\ D-97074 Würzburg, Germany \\ S. Hesselbach \\ Institut für Theoretische Physik, Universität Wien, A-1090 Wien, Austria
}

\begin{abstract}
Neutralinos with a large singlino component may appear in extended supersymmetric models with additional singlet Higgs fields. Since singlinos do not couple to (s)fermions and gauge bosons, the cross sections for the production of singlino dominated neutralinos are generally small. Within the framework of the Next-toMinimal Supersymmetric Standard Model (NMSSM) and an $\mathrm{E}_{6}$ inspired model we study neutralino production $e^{+} e^{-} \rightarrow \tilde{\chi}_{1}^{0} \tilde{\chi}_{2}^{0}\left(\tilde{\chi}_{1,2}^{0} \tilde{\chi}_{3}^{0}\right)$ with a singlino dominated $\tilde{\chi}_{2}^{0}$ $\left(\tilde{\chi}_{3}^{0}\right)$. It is shown that neutralinos with a singlino contribution up to $99 \%$ can be produced with a cross section larger than $1 \mathrm{fb}$ and may therefore be detected at a high luminosity $e^{+} e^{-}$linear collider even if they are not the LSP.
\end{abstract}

\section{Introduction}

The production of neutralinos, the supersymmetric partners of the neutral gauge and Higgs bosons, is expected to be among the most promising processes to discover a supersymmetric particle. As soon as a neutralino is detected a detailed study of its mass and mixing properties is necessary in order to deduce the underlying supersymmetric model. It has to be determined whether a neutralino can be assigned to the Minimal Supersymmetric Standard Model (MSSM) or belongs to an extension by additional singlet fields. A characteristic feature of extended supersymmetric models with singlet Higgs fields is the existence of neutralinos with large singlino components. Since, however, their couplings to (s)fermions and gauge bosons are strongly suppressed, it is often expected that only a singlino dominated neutralino which is the lightest supersymmetric particle (LSP) can be identified in extended models with $R$-parity conservation where it is in

\footnotetext{
*e-mail: fabian@physik.uni-wuerzburg.de

$\dagger$ e-mail: hesselb@thp.univie.ac.at
} 
the final state in all supersymmetric decay chains [1]. Especially within the framework of the Next-to-Minimal Supersymmetric Standard Model (NMSSM) it is argued that a singlino-like neutralino which is not the LSP could not be detected since it is not directly produced with a sufficient cross section and also omitted in the decay chains of the heavier supersymmetric particles.

Consequently, nonminimal neutralino production so far has only been discussed in supersymmetric scenarios where the lightest neutralino has a dominant singlet component and the other neutralinos have mainly MSSM properties [2, 3]. The aim of this study is a detailed analysis of the production cross section $e^{+} e^{-} \rightarrow \tilde{\chi}_{1}^{0} \tilde{\chi}_{2}^{0}\left(\tilde{\chi}_{1}^{0} \tilde{\chi}_{3}^{0}, \tilde{\chi}_{2}^{0} \tilde{\chi}_{3}^{0}\right)$, with a singlino dominated second (third) neutralino.

In Sec. 2 our scenarios in two representative extended supersymmetric models, the unconstrained NMSSM and an $\mathrm{E}_{6}$ model with an additional neutral gauge boson are described. A detailed analysis of the cross sections including the option of polarized electron and positron beams as a function of the singlino component of the produced neutralino follows in Sec. 3. In our conclusion we point out that, depending on the supersymmetric parameters and the selectron masses, cross sections up to $1 \mathrm{fb}$ can be reached even for neutralinos with a singlino content of $99 \%$. Therefore a high luminosity linear collider with polarized beams promises to be an excellent tool for the detection of singlino dominated non-LSP neutralinos and a discrimination between the minimal and extended supersymmetric models.

\section{Scenarios}

The production of a singlino dominated neutralino with a mass of $150 \mathrm{GeV}$ that is not the LSP at a linear $e^{+} e^{-}$collider is discussed within the framework of two extensions of the Minimal Supersymmetric Standard Model (MSSM) by a singlet Higgs field $S$.

\section{$2.1 \quad$ NMSSM}

In the unconstrained Next-to-Minimal Supersymmetric Standard Model (NMSSM) [ ] the masses and couplings of the five neutralinos depend on the the gaugino mass parameters $M_{2}$ and $M_{1}$, the ratio of the vacuum expectation values of the doublet Higgs fields $H_{1}$ and $H_{2}, \tan \beta=v_{2} / v_{1}$, the vacuum expectation value $x$ of the singlet Higgs field $S$ and the trilinear couplings $\lambda$ and $\kappa$ in the superpotential $W \supset \lambda H_{1} H_{2} S-1 / 3 \kappa S^{3}$ [5]. In our calculations we always assume the GUT relation $M_{1} / M_{2}=5 / 3 \tan ^{2} \theta_{W}$. For large $x \gg\left|M_{2}\right|$ a singlino dominated neutralino with mass eigenvalue $\approx 2 \kappa x$ in zeroth approximation decouples in the neutralino mixing matrix while the other neutralinos have MSSM character with $\mu=\lambda x$. The singlino content of the $i$-th neutralino $\tilde{\chi}_{i}^{0}$ is described by the squared matrix element $\left|N_{i 5}\right|^{2}$ of the unitary $5 \times 5$ matrix $N$ which diagonalizes the neutralino mass matrix in the basis $\left(\tilde{\gamma}, \tilde{Z}, \tilde{H}_{1}, \tilde{H}_{2}, \tilde{S}\right)$ of the photino, zino, the two doublet higgsinos and the singlino.

In the following section we analyze the cross sections for the production of a singlino dominated neutralino together with an MSSM-like $\tilde{\chi}_{1}^{0}$ or $\tilde{\chi}_{2}^{0}$ in representative scenarios where the LSP is mainly a gaugino or a higgsino with mass $100 \mathrm{GeV}$ (Table 1). The singlet 
vacuum expectation value $x$ is varied in order to study different singlino contents, but the mass of the singlino dominated neutralino which is the second lightest neutralino in the gaugino scenario NMSSM-G $\left(M_{2}=211 \mathrm{GeV}, \lambda x=400 \mathrm{GeV}\right)$ and the third lightest in the higgsino scenario NMSSM-H $\left(M_{2}=-400 \mathrm{GeV}, \lambda x=107 \mathrm{GeV}\right)$ is fixed at $150 \mathrm{GeV}$ by the parameter $\kappa$. Note that in the higgsino scenario both light neutralinos have higgsino character with different signs of the mass eigenvalues.

Fig. 11 shows the mixing character of the singlino dominated neutralinos. The singlino component crucially depends on the singlet vacuum expectation value $x$ [6]. In order to obtain a singlino content of $90 \%, x$ must be larger than $1200 \mathrm{GeV}$ (NMSSM-G) or $1000 \mathrm{GeV}$ (NMSSM-H). Generally the singlino component increases with increasing value of $x$.

A singlino content $\left|N_{25}\right|^{2}$ of $99 \%$ for $\tilde{\chi}_{2}^{0}$ with $m_{\tilde{\chi}_{2}^{0}}=150 \mathrm{GeV}$ is reached at $x=$ $3750 \mathrm{GeV}, \kappa=0.02$ in the gaugino scenario. In the higgsino scenario $\tilde{\chi}_{3}^{0}$ is a $99 \%$ singlino for $x=2750 \mathrm{GeV}$ and $\kappa=0.0275$ (Table 1 ). In section 3 these values will be used to compute production cross sections.

The couplings of the singlino dominated neutralino and therefore its production cross sections are determined by the remaining MSSM components. It is the gaugino component that dominates the MSSM content in the scenario NMSSM-G with a gaugino-like LSP while in the scenario NMSSM-H with a higgsino-like LSP the MSSM-higgsino component prevails. These mixing characteristics facilitate the identification of the production of the singlino-like neutralino since it enhances the corresponding cross sections for the associated production of the light neutralinos (Sec. 3).

\section{$2.2 \quad \mathrm{E}_{6}$ model}

We consider an $\mathrm{E}_{6}$ model with one extra neutral gauge boson $Z^{\prime}$ and one additional singlet superfield [7]. To respect the experimental mass bounds for new gauge bosons the singlet vacuum expectation value $x$ must be larger than about $1.5 \mathrm{TeV}$ [8]. The extended neutralino sector in this model contains six neutralinos being mixtures of photino, zino, doublet higgsinos, $Z^{\prime}$ gaugino $\left(\tilde{Z}^{\prime}\right)$, and singlino [9, 10]. The $6 \times 6$ neutralino mass matrix depends on six parameters: the $\mathrm{SU}(2)_{\mathrm{L}}, \mathrm{U}(1)_{Y}$ and $\mathrm{U}(1)^{\prime}$ gaugino mass parameters $M_{2}$, $M_{1}$ and $M^{\prime}, \tan \beta, x$ and the trilinear coupling $\lambda$ in the superpotential $W \supset \lambda H_{1} H_{2} S$. Analogously to the NMSSM, the matrix element $\left|N_{i 6}\right|^{2}$ of the unitary $6 \times 6$ matrix that diagonalizes the neutralino mass matrix describes the singlino content of the $i$-th neutralino in the basis $\left(\tilde{\gamma}, \tilde{Z}, \tilde{H}_{1}, \tilde{H}_{2}, \tilde{Z}^{\prime}, \tilde{S}\right)$.

In the $\mathrm{E}_{6}$ model the $4 \times 4$ submatrix of the MSSM-like neutralinos and the $2 \times 2$ submatrix of the exotic ones approximately decouple because of the large values of $x$. For very large values of $\left|M^{\prime}\right| \gg x$ a neutralino with a mass of $150 \mathrm{GeV}$ can be a nearly pure singlino [11] with mass $\approx 0.18 x^{2} /\left|M^{\prime}\right|$ in zeroth approximation.

As in the NMSSM we choose scenarios where the lightest neutralino with a mass of $100 \mathrm{GeV}$ is mainly a gaugino or a higgsino (Table 1$)$. The singlino-like neutralino $\left(\tilde{\chi}_{2}^{0}\right.$ in the gaugino scenarios and $\tilde{\chi}_{3}^{0}$ in the higgsino scenarios) can be fixed at $150 \mathrm{GeV}$ by choosing an appropriate value for $M^{\prime}$ even if the singlet vacuum expectation value is varied. For positive $M^{\prime}$ the singlino dominated neutralino has opposite sign of the mass eigenvalue relative to the LSP than in the NMSSM, while for $M^{\prime}<0$ the mass eigenvalue 


\begin{tabular}{|c|c|c|c|c|c|c|}
\hline Scenario & NMSSM-G & NMSSM-H & $\mathrm{E}_{6}-\mathrm{G}+$ & $\mathrm{E}_{6}-\mathrm{G}-$ & $\mathrm{E}_{6}-\mathrm{H}+$ & $\mathrm{E}_{6}-\mathrm{H}-$ \\
\hline \hline$M_{2} / \mathrm{GeV}$ & 211 & -400 & 211 & 211 & -400 & -400 \\
$\lambda x / \mathrm{GeV}$ & 400 & 107 & 400 & 400 & 107 & 107 \\
$\tan \beta$ & 3 & 3 & 3 & 3 & 3 & 3 \\
$\tilde{\chi}_{1}^{0}$ character & gaugino & higgsino & gaugino & gaugino & higgsino & higgsino \\
$m_{\tilde{\chi}_{1}} / \mathrm{GeV}$ & $(+) 100$ & $(+) 100$ & $(+) 100$ & $(+) 100$ & $(+) 100$ & $(+) 100$ \\
$m_{\tilde{\chi}_{2}} / \mathrm{GeV}$ & $(+) 150$ & $(-) 122$ & $(-) 150$ & $(+) 150$ & $(-) 122$ & $(-) 122$ \\
$m_{\tilde{\chi}_{3}} / \mathrm{GeV}$ & $(+) 190$ & $(-) 150$ & $(+) 190$ & $(+) 190$ & $(+) 150$ & $(-) 150$ \\
\hline \hline$x / \mathrm{GeV}$ & 3750 & 2750 & 4100 & 4700 & 3700 & 4200 \\
$m_{Z^{\prime}} / \mathrm{GeV}$ & - & - & 1726 & 1979 & 1558 & 1768 \\
$\kappa$ & 0.02 & 0.0272 & - & - & - & - \\
$M^{\prime}$ & - & - & 19652.4 & -25979.6 & 16015.4 & -20704.8 \\
$\left|N_{25}\right|^{2}$ & 0.99 & & 0.99 & 0.99 & & \\
$\left|N_{35}\right|^{2}$ & \multicolumn{7}{|l}{} & 0.99 & & & 0.99 & 0.99 \\
\hline \hline
\end{tabular}

Table 1: Parameters of the supersymmetric models in representative scenarios. The mass of the singlino dominated neutralino is fixed by the parameters $\kappa$ (NMSSM) and $M^{\prime}\left(\mathrm{E}_{6}\right.$ model). The signs in parentheses show the relative sign of the neutralino mass eigenvalues in comparison to the mass eigenvalues of the lightest neutralino.

of this neutralino flips sign and thus becomes the same as in the NMSSM. Therefore the properties of the singlino-like neutralino in the $\mathrm{E}_{6}$ scenarios $\mathrm{E}_{6}-\mathrm{G}-$ and $\mathrm{E}_{6}-\mathrm{H}$ - with negative $M^{\prime}$ are more similar to those in the NMSSM.

The MSSM components of the singlino dominated neutralino in the $\mathrm{E}_{6}$ scenarios are, however, significantly smaller than the $\tilde{Z}^{\prime}$ components and also than the MSSM components in the NMSSM. This mixing characteristic leads to smaller production cross sections compared to the NMSSM as discussed in the next section. Contrary to the NMSSM, in the scenario $\mathrm{E}_{6}-\mathrm{G}+$ with a gaugino-like LSP the MSSM doublet higgsino component even outweighs the MSSM gaugino component which results in a further suppression of the production cross section in this scenario.

As in the NMSSM the singlino content increases also in the $\mathrm{E}_{6}$ model with increasing vacuum expectation value $x$ (Fig. (1). In order to obtain a singlino content exceeding $99 \%$ the singlet vacuum expectation value $x$ must be larger than about $3.5-4 \mathrm{TeV}$ in our scenarios.

\section{Cross sections}

We study the associated production of a singlino dominated neutralino $e^{+} e^{-} \rightarrow \tilde{\chi}_{1}^{0} \tilde{\chi}_{2}^{0}$ in the scenarios with a gaugino-like LSP and $e^{+} e^{-} \rightarrow \tilde{\chi}_{1,2}^{0} \tilde{\chi}_{3}^{0}$ in the scenarios where the 

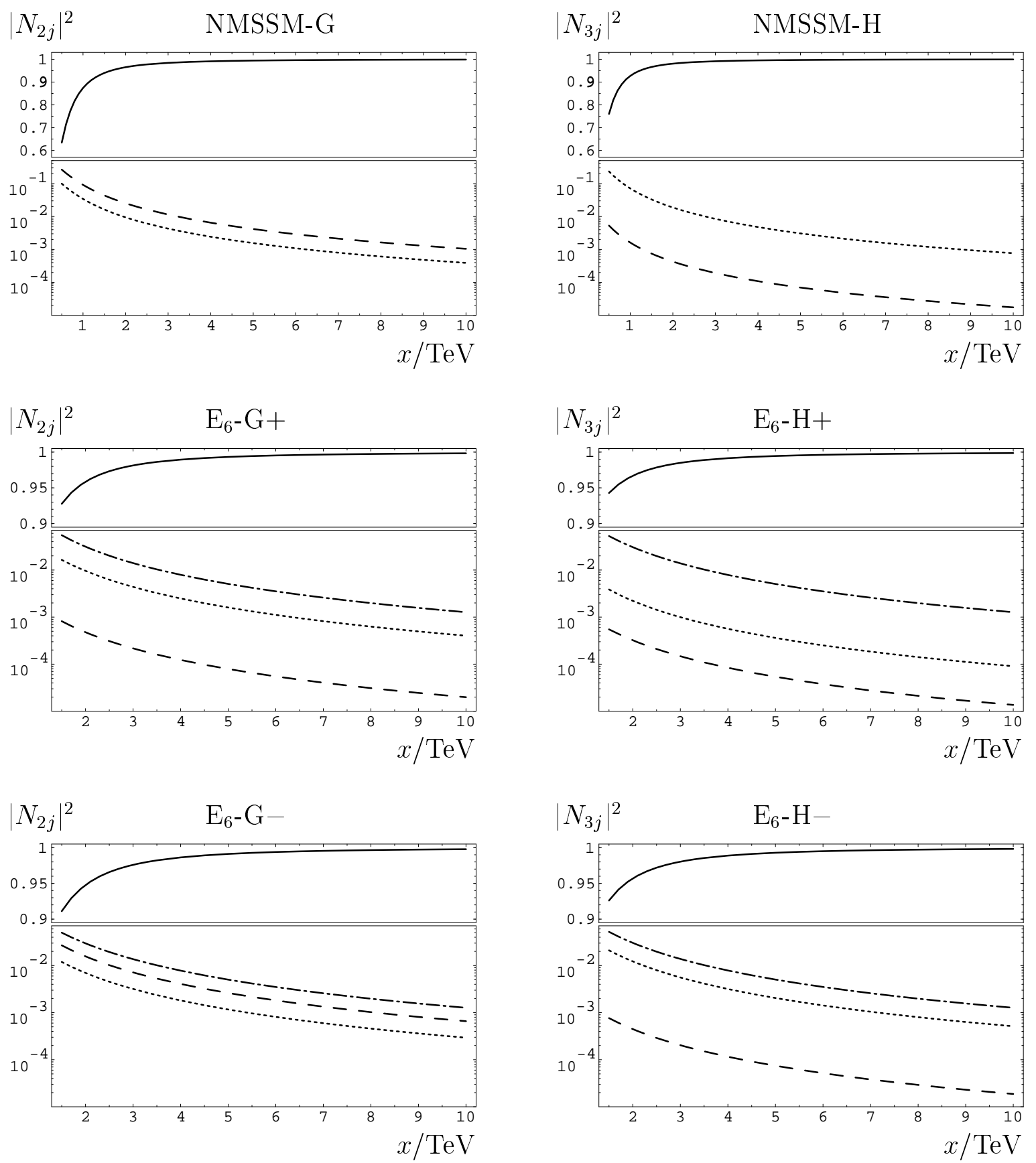

Figure 1: Mixing of the singlino dominated neutralino in the scenarios of Table 1: singlino component (solid line), MSSM gaugino components $\left(\left|N_{i 1}\right|^{2}+\left|N_{i 2}\right|^{2}\right.$, dashed), MSSM doublet higgsino components $\left(\left|N_{i 3}\right|^{2}+\left|N_{i 4}\right|^{2}\right.$, dotted) and $\mathrm{E}_{6} \tilde{Z}^{\prime}$ component (dashed-dotted). The mass of the singlino dominated neutralino is fixed at $150 \mathrm{GeV}$ by the parameters $\kappa$ (NMSSM) and $M^{\prime}\left(\mathrm{E}_{6}\right.$ model). 
lightest and second lightest neutralinos are mainly higgsinos. Neutralino production in $e^{+} e^{-}$annihilation proceeds via the exchange of a $Z$ boson in the $s$ channel and of left and right selectrons in the $t$ and $u$ channel. In the $\mathrm{E}_{6}$ model the contribution of the additional $Z^{\prime}$ boson has to be considered. Due to the large $Z^{\prime}$ mass in the scenarios of Table 1, however, $Z^{\prime}$ resonance effects appear far beyond expected energies of a linear collider at first stages. Since the singlino component does not couple to gauge bosons and fermions or sfermions due to the hypercharge 0 of the singlet field, the cross section for the production of neutralinos with a significant singlino component is strongly suppressed.

Analytical formulae are given in [12 for the NMMSM and [10 for the $\mathrm{E}_{6}$ model. For comparison, the masses of the left and right selectrons $m_{\tilde{e}_{L}}=300 \mathrm{GeV}, m_{\tilde{e}_{R}}=200 \mathrm{GeV}$ are fixed in all scenarios.

The use of polarized beams may be an excellent tool to enhance cross sections and to identify the supersymmetric signal. Polarizations up to $85 \%$ for electrons and $60 \%$ for positrons are expected at a future linear collider [13]. We consider the polarization configurations $P_{-}=+0.85, P_{+}=-0.6$ (right polarized electrons and left polarized positrons) and $P_{-}=-0.85, P_{+}=+0.6$ (left polarized electrons and right polarized positrons) in our studies.

In the following we assume a cross section of $1 \mathrm{fb}$ to be sufficient for the identification of a neutralino production process. Of course the discovery limit depends on the neutralino decay properties that are discussed in detail in [1, 6, 12,

In Fig. 2 the cross sections for the production of a neutralino with a singlino content of $99 \%$ are shown for beam energies between the threshold and $2 \mathrm{TeV}$ in the NMSSM scenarios of Table 1. For unpolarized beams the cross section for $\tilde{\chi}_{1}^{0} \tilde{\chi}_{2}^{0}$ production reaches values up to $1 \mathrm{fb}$ in the gaugino scenario NMSSM-G with selectron exchange being the dominant channel.

In the higgsino scenario NMSSM-H neutralino production proceeds mainly by $Z$ boson exchange. Then the cross sections for $e^{+} e^{-} \rightarrow \tilde{\chi}_{1,2}^{0} \tilde{\chi}_{3}^{0}$ of the production of a singlino dominant $\tilde{\chi}_{3}^{0}$ depend on the MSSM-higgsino content of $\tilde{\chi}_{3}^{0}$ and the $Z$-higgsino coupling. While $\tilde{\chi}_{1}^{0} \tilde{\chi}_{3}^{0}$ production reaches unpolarized cross sections up to $5 \mathrm{fb}$, the cross section for $\tilde{\chi}_{2}^{0} \tilde{\chi}_{3}^{0}$ is nearly two orders of magnitude smaller and significantly below discovery limit. In the latter case the ratio of the doublet higgsino components $N_{33} / N_{34}$ of $\tilde{\chi}_{3}^{0}$ is similar to $N_{23} / N_{24}$ of $\tilde{\chi}_{2}^{0}$ which results in a small $Z$-higgsino coupling because higgsino pair production is generally suppressed.

The unpolarized cross section can be significantly enhanced by a factor of $2-3$ in all scenarios by choosing a suitable beam polarization. In the gaugino scenario NMSSM-G where the neutralinos $\tilde{\chi}_{1}^{0}$ and $\tilde{\chi}_{2}^{0}$ couple preferably to the right selectron, the polarization configuration $P_{-}>0, P_{+}<0$ enlarges the cross sections, while in the higgsino scenario the cross sections are largest for $P_{-}<0, P_{+}>0$ [3, 14].

Fig. 2 also shows the cross section for the associated neutralino production at a linear collider with a center-of-mass energy of $500 \mathrm{GeV}$ as a function of the singlino content. For the associated production of the second lightest neutralino which is a $90 \%$ singlino and a gaugino-dominated LSP in scenario NMSSM-G one obtains cross sections of $15 \mathrm{fb}$ with unpolarized beams or $40 \mathrm{fb}$ with beam configuration $P_{-}=+0.85, P_{+}=-0.6$. In scenario NMSSM-H the third lightest neutralino with a singlino content of $90 \%$ and the higgsinodominated LSP are produced with $20 \mathrm{fb}$ (unpolarized beams) and $40 \mathrm{fb}\left(P_{-}=-0.85\right.$, 
$\left.P_{+}=+0.6\right)$ in the process $e^{+} e^{-} \rightarrow \tilde{\chi}_{1}^{0} \tilde{\chi}_{3}^{0}$. Assuming a discovery limit of $1 \mathrm{fb}$, a neutralino with a singlino content of $99.2 \%$ can be detected at a linear collider with unpolarized beams in the gaugino scenario and even with a singlino content of $99.5 \%$ in the higgsino scenario although it is not the LSP.

In Fig. 3 we show the corresponding cross sections in the $\mathrm{E}_{6}$ model for the associated production of a singlino-dominated neutralino in the $\mathrm{E}_{6}$ scenarios of Table 1 . In the $\mathrm{E}_{6}$ model the singlino-dominated neutralino also has a considerable $\tilde{Z}^{\prime}$ component which even is the second largest component. It couples to the selectrons exchanged in $t$ and $u$ channels [10] but nevertheless contributes significantly less to the cross section than the doublet higgsino and gaugino components. Therefore the cross sections for the associated production of a singlino-dominated neutralino are in the $\mathrm{E}_{6}$ model for energies below the $Z^{\prime}$ resonance generally smaller than in the NMSSM.

In scenario $\mathrm{E}_{6}-\mathrm{G}+$ both the singlino-dominated $\tilde{\chi}_{2}^{0}$ and the gaugino-like LSP couple preferably to the right selectron. The unpolarized cross section for the associated production of $\tilde{\chi}_{2}^{0}$ with a singlino content of $99 \%$ reaches a maximum value of $0.4 \mathrm{fb}$ for $\sqrt{s}<1500 \mathrm{GeV}$ which is enhanced to $1.2 \mathrm{fb}$ for beam polarization $P_{-}=+0.85, P_{+}=-0.6$. Cross sections of $1 \mathrm{fb}$ at a linear collider with $\sqrt{s}=500 \mathrm{GeV}$ are obtained for the associated production of a neutralino with a singlino content as high as $97 \%$ (unpolarized beams) or $99 \%\left(P_{-}=+0.85, P_{+}=-0.6\right)$.

In scenario $\mathrm{E}_{6}-\mathrm{G}-$, however, the singlino-dominated $\tilde{\chi}_{2}^{0}$ couples mainly to the heavier left selectron contrary to $\tilde{\chi}_{1}^{0}$. Hence the cross sections are considerably smaller with only $0.07 \mathrm{fb}$ at $\sqrt{s}=500 \mathrm{GeV}$ for unpolarized beams. Here a second lightest neutralino with a singlet component smaller than $93 \%$ is produced with a cross section of at least $1 \mathrm{fb}$ at a $500 \mathrm{GeV}$ linear collider with beam polarization $P_{-}=+0.85, P_{+}=-0.6$. Due to the increased contribution of the exchange of the left selectron it is the opposite polarization configuration $P_{-}=-0.85, P_{+}=+0.6$ compared to scenario $\mathrm{E}_{6}$ - $\mathrm{G}+$ that enhances the cross section for larger singlino components.

As to the $\mathrm{E}_{6}$ higgsino scenarios only cross sections for the process $e^{+} e^{-} \rightarrow \tilde{\chi}_{1}^{0} \tilde{\chi}_{3}^{0}$ in scenario $\mathrm{E}_{6}-\mathrm{H}-$ are given in Fig. 3. In scenario $\mathrm{E}_{6}-\mathrm{H}+$ the cross sections for the associated production of the singlino-dominated $\tilde{\chi}_{3}^{0}$ are strongly suppressed since its doublet higgsino content is more than one order of magnitude smaller than in the NMSSM. Similar to the NMSSM also the $\tilde{\chi}_{2}^{0} \tilde{\chi}_{3}^{0}$ production in scenario $\mathrm{E}_{6}-\mathrm{H}-$ is invisible. However, the unpolarized cross section for $\tilde{\chi}_{1}^{0} \tilde{\chi}_{3}^{0}$ production at a linear collider reaches $0.7 \mathrm{fb}$ outside the $Z^{\prime}$ resonance and is enhanced to $1.4 \mathrm{fb}$ with beam polarization $P_{-}=-0.85, P_{+}=+0.6$.

Assuming a discovery limit of $1 \mathrm{fb}$, a neutralino with a singlino content up to $96.5 \%$ (unpolarized beams) or even up to $98.2 \%\left(P_{-}=-0.85, P_{+}=+0.6\right)$ can be detected at a linear collider with $\sqrt{s}=500 \mathrm{GeV}$ in the higgsino scenario $\mathrm{E}_{6}-\mathrm{H}-$.

Generally the beam polarization configurations which enhance the cross sections in gaugino and higgsino scenarios in the $\mathrm{E}_{6}$ model outside the $Z^{\prime}$ resonance are independent of the energy or the singlino content. In the vicinity of the $Z^{\prime}$ resonance, however, resonance effects radically change the polarization asymmetry. In our scenarios these effects are out of reach at the first stages of a linear collider, but appear at $\sqrt{s}=500 \mathrm{GeV}$ for a singlino content less than $96 \%$ in the scenario $\mathrm{E}_{6}-\mathrm{G}-$ and $97 \%$ in $\mathrm{E}_{6}-\mathrm{H}-$, where the $Z^{\prime}$ boson becomes rather light with a mass smaller than $1 \mathrm{TeV}$. 

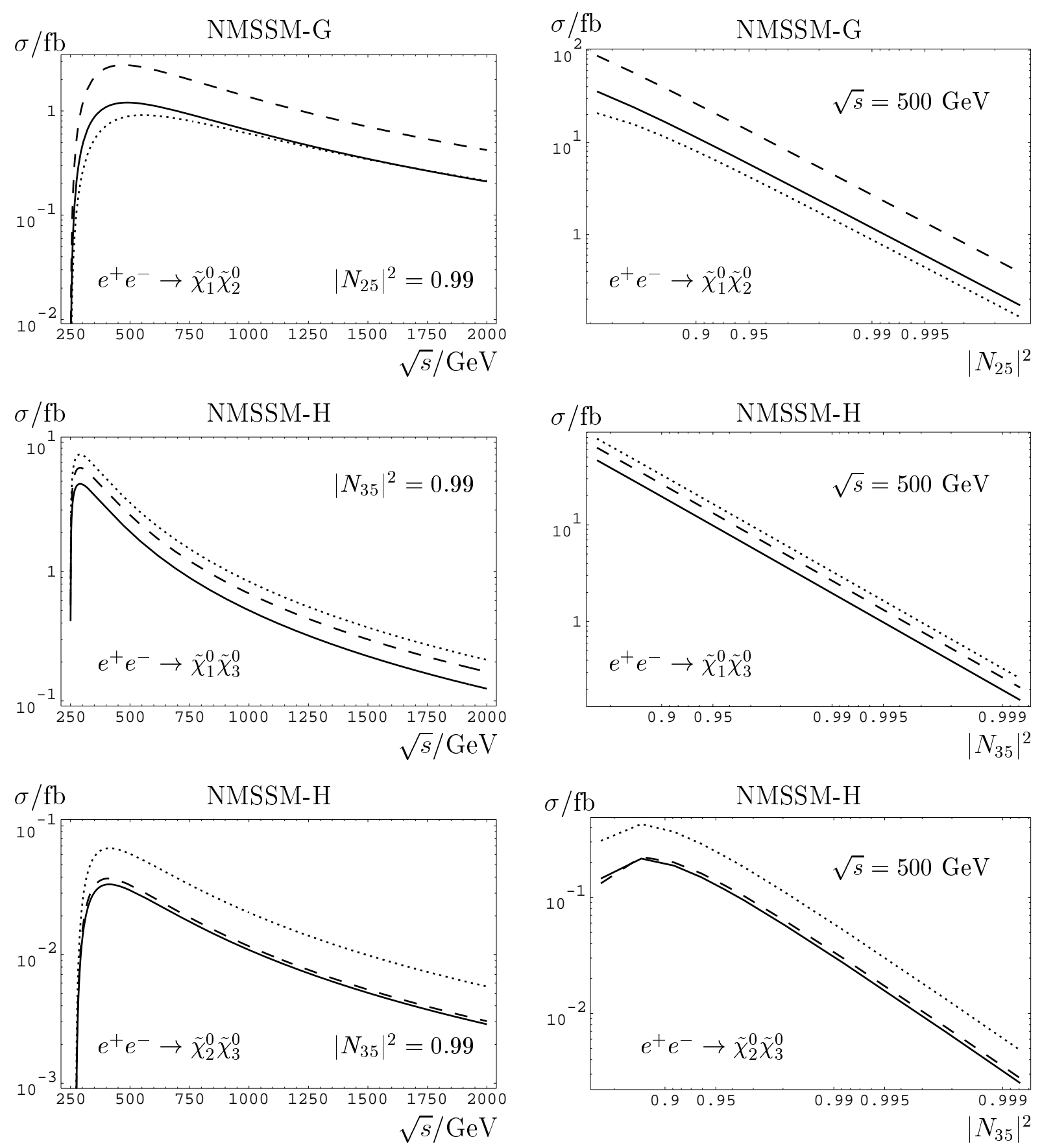

Figure 2: Cross sections for the production of a singlino dominated neutralino in the NMSSM scenarios given in Table 1 with unpolarized beams (solid) and beam polarizations $P_{-}=+0.85, P_{+}=-0.6$ (dashed) and $P_{-}=-0.85, P_{+}=+0.6$ (dotted). In the plots on the right hand side the singlet vacuum expectation value $x$ is varied and the mass of the singlino dominated neutralino is fixed at $150 \mathrm{GeV}$ by the parameter $\kappa$. 

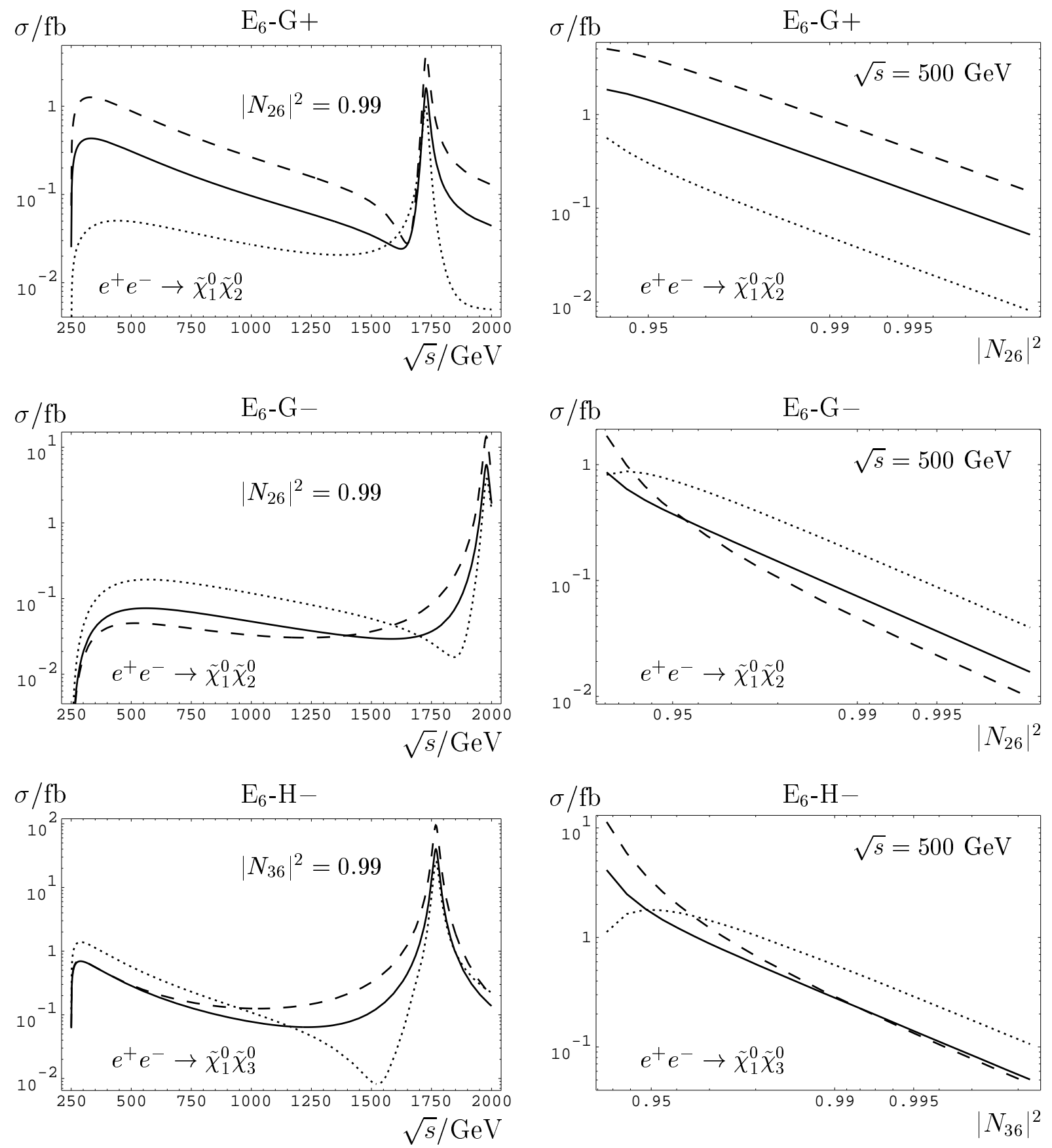

Figure 3: Cross sections for the production of a singlino dominated neutralino in the $\mathrm{E}_{6}$ model scenarios given in Table 1 with unpolarized beams (solid) and beam polarizations $P_{-}=+0.85, P_{+}=-0.6$ (dashed) and $P_{-}=-0.85, P_{+}=+0.6$ (dotted). In the plots on the right hand side the singlet vacuum expectation value $x$ is varied and the mass of the singlino dominated neutralino is fixed at $150 \mathrm{GeV}$ by the parameter $M^{\prime}$. 


\section{Conclusion}

Experimental evidence of a neutralino with a significant singlino component would be an explicit proof for an extended supersymmetric model with additional Higgs singlet fields. A high luminosity $e^{+} e^{-}$linear collider offers the opportunity to study the associated production of a singlino-dominated neutralino. Complementary to existing analyses we have shown that assuming a discovery limit of $1 \mathrm{fb}$ a neutralino with a singlino content larger than $90 \%$ can be detected at a linear collider although it is not the LSP. The use of suitably polarized beams enhances the production cross sections and facilitates the identification of the singlino-dominant neutralino.

In representative scenarios with a gaugino-like LSP one obtains unpolarized cross sections of $1 \mathrm{fb}$ for the associated production $e^{+} e^{-} \rightarrow \tilde{\chi}_{1}^{0} \tilde{\chi}_{2}^{0}$ of a second lightest neutralino with a singlino content of $99.2 \%$ in the NMSSM and $97 \%$ in the $\mathrm{E}_{6}$ model at a linear collider with $500 \mathrm{GeV}$ center-of-mass energy. Due to the additional $\tilde{Z}^{\prime}$ component of the neutralinos, cross sections are generally smaller in the $\mathrm{E}_{6}$ model below the $Z^{\prime}$ resonance. In gaugino scenarios mainly the exchange of left and right selectrons contribute to the cross section. If the coupling to right (left) selectrons is dominant the beam polarization $P_{-}>0, P_{+}<0\left(P_{-}<0, P_{+}>0\right)$ increases the cross section by a factor of $2-3$.

Also in scenarios where both light neutralinos have higgsino character the cross sections for the associated production of a singlino-dominated third neutralino exceed the discovery limit. Here it is the polarization configuration $P_{-}<0, P_{+}>0$ that enhances the unpolarized cross sections.

Light neutralinos with a large singlet component may exist in extended supersymmetric models, but not necessarily as the LSP. Even then discrimination between supersymmetric models by the direct production of a singlino-dominated neutralino is a realistic goal at a future high luminosity electron-positron linear collider.

\section{Acknowledgment}

We thank A. Bartl and H. Fraas for many helpful discussions and the careful reading of the manuscript. This work is supported by the 'Fonds zur Förderung der wissenschaftlichen Forschung' of Austria, FWF Project No. P13139-PHY and by the EU TMR Project No. HPRN-CT-2000-00149. S.H. is supported by the Deutsche Forschungsgemeinschaft (DFG) under contract No. HE 3241/1-1.

\section{References}

[1] U. Ellwanger and C. Hugonie, Eur. Phys. J. C 5 (1998) 723; Eur. Phys. J. C 13 (2000) 681.

[2] G. Moortgat-Pick, S. Hesselbach, F. Franke and H. Fraas, WUE-ITP-99-023, hepph/9909549, contribution to the Proceedings of the 4 th International Workshop on Linear Colliders (LCWS99), Sitges, Barcelona, Spain, April 28 - May 5, 1999. 
[3] S. Hesselbach, F. Franke and H. Fraas, in Physics and Experimentation at a Linear Electron-Positron Collider, Contributions to the 2nd ECFA/DESY Study, 1998 2001, Ed. T. Behnke, S. Bertolucci, R.D. Heuer, D. Miller, F. Richard, R. Settles, V. Telnov, P. Zerwas (DESY 01-123F, Hamburg, 2001) p. 753.

[4] H.P. Nilles, M. Srednicki and D. Wyler, Phys. Lett. B 120 (1983) 346;

J.-P. Derendinger and C.A. Savoy, Nucl. Phys. B 237 (1984) 307;

M. Drees, Int. J. of Mod. Phys. A 4 (1989) 3635;

J. Ellis, J.F. Gunion, H.E. Haber, L. Roszkowski and F. Zwirner, Phys. Rev. D 39 (1989) 844;

T. Elliot, S.F. King and P.L. White, Phys. Lett. B 314 (1993) 56; Phys. Rev. D 49 (1994) 2435;

U. Ellwanger, M. Rausch de Traubenberg and C.A. Savoy, Phys. Lett. B 315 (1993) 331 ;

B.R. Kim, A. Stephan and S.K. Oh, Phys. Lett. B 336 (1994) 200.

[5] F. Franke and H. Fraas, Int. J. Mod. Phys. A12 (1997) 479;

F. Franke, H. Fraas and A. Bartl, Phys. Lett. B 336 (1994) 415.

[6] S. Hesselbach, F. Franke and H. Fraas, Phys. Lett. B 492 (2000) 140.

[7] J.F. Gunion, L. Roszkowski and H.E. Haber, Phys. Lett. B 189 (1987) 409; Phys. Rev. D 38 (1988) 105;

M. Drees, Nucl. Phys. B 298 (1988) 333;

J.L. Hewett and T.G. Rizzo, Phys. Rep. 183 (1989) 193;

M.M. Boyce, M.A. Doncheski and H. König, Phys. Rev. D 55 (1997) 68;

M. Cvetič, D.A. Demir, J.R. Espinosa, L. Everett and P. Langacker, Phys. Rev. D 56 (1997) 2861; Phys. Rev. D 58 (1998) 119905(E);

M. Cvetič and P. Langacker, in Perspectives in Supersymmetry, Ed. G.L. Kane (World Scientific), p. 312;

T. Gherghetta, T.A. Kaeding and G.L. Kane, Phys. Rev. D 57 (1998) 3178;

P. Langacker and J. Wang, Phys. Rev. D 58 (1998) 115010;

L. Everett, P. Langacker, M. Plümacher and J. Wang, Phys. Lett. B 477 (2000) 233.

[8] F. Abe et al. (CDF Collaboration), Phys. Rev. Lett. 79 (1997) 2192.

[9] J. Ellis, K. Enqvist, D.V. Nanopoulos and F. Zwirner, Nucl. Phys. B 276 (1986) 14;

S. Nandi, Phys. Lett. B 197 (1987) 144;

E. Keith and E. Ma, Phys. Rev. D 54 (1996) 3587; Phys. Rev. D 56 (1997) 7155;

D. Suematsu, Mod. Phys. Lett A 12 (1997) 1709; Phys. Lett. B 416 (1998) 108;

Phys. Rev. D 57 (1998) 1738.

[10] S. Hesselbach, F. Franke and H. Fraas, MADPH-01-1237, hep-ph/0107080.

[11] B. de Carlos and J.R. Espinosa, Phys. Lett. B 407 (1997) 12.

[12] F. Franke and H. Fraas, Z. Phys. C 72 (1996) 309. 
[13] G. Moortgat-Pick and H. Steiner, Eur. Phys. J. direct C 6 (2001) 1.

[14] G. Moortgat-Pick, A. Bartl, H. Fraas and W. Majerotto, Eur. Phys. J. C 18 (2000) 379. 